\title{
Sub-pathway based approach to systematically track candidate sub-pathway biomarkers for heart failure
}

\author{
DONG-MEI HAN
}

\begin{abstract}
Department of Cardiology, General Hospital of Daqing Oil Field, Daqing, Heilongjiang 163000, P.R. China
\end{abstract}
Received June 18, 2018; Accepted February 6, 2019

DOI: 10.3892/etm.2019.7319

\begin{abstract}
Identification of potential novel biomarkers for heart failure was undertaken using a sub-pathway based method. To realize this goal, heart failure-relevant dataset, reference pathways, and IncRNA-miRNA-mRNA interactions were firstly recruited. Secondly, the informative pathways were extracted relying on KEGG pathways and the mRNAs in the PCC-weighted IncRNA-mRNA interactions. Thirdly, lncRNA-regulated sub-pathways were dissected after construction of condition-specific lncRNA competitively regulated pathways (LCRP). To detect crucial heart failure-relevant lncRNAs, degree analysis was conducted for all nodes within the LCRP. Ultimately, the significance of candidate sub-pathways were assessed to further identify the significant sub-pathways. There were 44 lncRNAs, 165 mRNAs and 224 co-expressed interactions. After putting the 165 mRNAs into the reference pathways, 56 informative pathways were obtained which were then embedded into undirected graphs, and 44 lncRNAs were inserted into the pathway graphs to further construct the condition-specific LCRP. According to degree distribution, 4 hub lncRNAs were selected, including ERVK13-1, YLPM1, PDXDC2P, and LINC00482. Based on the LCRP information, a total of 36 sub-pathways mediated by lncRNAs participated in 40 complete pathways. Among these 40 pathways, we mainly concentrated on the top three sub-pathways, including a sub-part of MAPK signaling pathway, an important sub-part in ErbB signaling pathway, and a part of chemokine signaling pathway. In the top 3 significant sub-pathways, gene AKT3 was simultaneously regulated by ERVK13-1, YLPM1, and PDXDC2P. Sub-pathways including MAPK signaling pathway and hub lncRNAs (ERVK13-1, YLPM1, and PDXDC2P) may play an important role in heart failure.
\end{abstract}

Correspondence to: Dr Dong-Mei Han, Department of Cardiology, General Hospital of Daqing Oil Field, 9 Zhongkang Street, Saertu, Daqing, Heilongjiang 163000, P.R. China

E-mail: daqingdong25@163.com

Key words: heart failure, condition-specific lncRNA competitively regulated pathways, subpathway

\section{Introduction}

Heart failure is a costly, and fatal health problem in the world (1). In 2015, this disease affected approximately 40 million people in the world (2). Approximately $50 \%$ of the heart failure patients die in 5 years after diagnosis, but more than half of the advanced heart failure patients die within the first year following diagnosis (3). The cost to health-care of heart failure is up to 30 billion dollars per year in US alone. According to the statistical data, an urgent need is highlighted for identifying more accurate and available heart failure signatures with diagnostic and prognostic values.

Identification of gene signatures for complicated diseases such as heart failure and cancer is greatly important for diagnosis, prognosis, as well as developing personalized medicine and treatment strategy. In recent years, an increasing number of studies have proposed many biomarkers associated with heart failure. For example, cardiac troponin T, a biomarker of cardiomyocyte injury, has been demonstrated to be a predictor of side effects for heart failure patients (4). Moreover, Kusumoto et al (4) demonstrated that MYH7, ANXA2, IGFBP7, as well as DESM are promising candidate signatures of heart failure both in human and mouse. Frequently, these biomarkers cannot reflect the substrate relationship between genotypes and phenotypes, therefore they offer little information in the etiology of disorders, resulting in the urgent need of elucidating the potential pathogenesis of the disease as well as developing treatments. Additionally, reliable and simple measurements to detect the disorder earlier as well as to efficiently predict the prognosis still remain insufficient.

Thus, detecting novel biomarkers is of critical importance to understand and prevent heart failure. Long non-coding RNAs (lncRNAs) are a class of ncRNAs with $>200$ nucleotides (5). LncRNAs have been implicated to play an important role, such as cell cycle, differentiation, and apoptosis $(6,7)$. Moreover, IncRNAs have been reported to competitively regulate biological pathways and to be closely related to heartrelated diseases, for example, heart failure, and hypertrophy (8-10). Therefore, it is necessary to integratively analyze the joint effect of lncRNAs and pathway topologies. Moreover, extracting pathways competitively regulated by lncRNAs not only can provide novel insights into the potential mechanisms, but also help us to explore the functions of lncRNAs in a given disease (11). Currently, the functions of lncRNAs under a given disease condition have not been well revealed and a method for 
uncovering the lncRNA role is needed. Fortunately, key local subregions, not complete pathways, have been proposed to be more explainable to the molecular mechanisms of diseases $(12,13)$. It is noteworthy that detecting the sub-pathways instead of entire pathways might be more relevant for interpreting the related biological meaning and extracting the functions of lncRNAs. No report on lncRNA-modulated sub-pathways in heart failure patients is available.

In this study, to explore the etiology of heart failure, the expression of IncRNA-mRNA and pathway topologies were profiled to extract the IncRNAs competitively regulated subpathways in heart failure using the sub-pathway based strategy.

\section{Materials and methods}

Gene expression data acquisition and pretreating. The gene expression profile on heart failure with the accession number E-GEOD-26887 (14) was recruited from Arrayexpress database, which was deposited in the platform of A-Affy141-Affymetrix GeneChip Human Gene 1.0 AT Array [HuGene-1-0-st-v1]. In the E-GEOD-26887 (14), there were 19 patients with heart failure, and 5 controls from left ventricle cardiac biopsies. In the present work, a total of 24 subjects were used for subsequent analysis.

Then, pre-treatment for the original microarray profile was conducted. The specific steps contained background correction using robust multiarray average (RMA) approach (15), normalization by means of quartile (16), probe correction via MAS, and summarization of probe values through Medianpolish method. After probes were transformed to the human gene symbols, the final gene expression matrix (covering 18,715 genes) was established.

Proposed scheme. Sub-pathway based method was used to detect sub-pathways competitively regulated by lncRNA in heart failure. This method included four steps: i) collection of candidate lncRNA-mRNA interactions; ii) using KEGG pathways as backbone, construction of condition-specific lncRNA competitively regulated pathways (LCRP) on the basis of lncRNA data, mRNA expression data, and lncRNAmRNA interactions; iii) aligning competing lncRNAs and the interesting genes into the condition-specific LCRP, then locating sub-pathways in pathways and iv) evaluation of the significance of candidate sub-pathways.

Collection of lncRNA-mRNA interactions. Firstly, lncRNAmiRNA interactions were recruited from StarBase database (version 2.0) (17), and mRNA-miRNA interactions were collected from the databases of mir2Disease, mirTarBase, TarBase, and miRecords (V4.0). On the basis of the common miRNAs, we collected candidate IncRNA-mRNA competitively regulated relationships. With the goal of guaranteeing data reliability, we filtered and reserved several candidate competing mRNAs for each lncRNA when they met the following criteria. The first criterion was that we utilized hypergeometric test to estimate the importance of the common miRNAs, and that the threshold of false discovery rate (FDR) was defined as 0.05 . The second criterion was that Jaccard Coefficient of the common miRNAs were ordered at top $20 \%$. Using these criteria, 7,693 candidate lncRNA-mRNA interactions covering 835 lncRNAs, and 1,749 mRNAs were obtained.

After that, we took the intersection between 18,715 genes of the microarray profile and 7,693 lncRNAs-mRNA relationships, we got a total of 1,605 mRNAs as well as 94 lncRNAs.

Co-expression analysis for the lncRNA-mRNA interactions using Pearson correlation coefficient (PCC). As known, PCC is an index of correlation between two variables, which ranges from -1 to 1 (18). Hence, in our study, PCC was used to assess the co-expression possibility for any pair of IncRNAmRNA interactions on the basis of the matched lncRNA and mRNA data. After analysis using Fisher's r-to-Z transformation (19), those IncRNA-mRNA interactions that possess $r$ value reaching a significant positive threshold were reserved $(\mathrm{P}<0.05)$.

Identification of informative pathways. KEGG is a database, which can provide reference knowledge to comprehensively understand cellular processes using pathway aligning (20). Thus, in our study, we downloaded all the KEGG background pathways based on KEGG database. After that, the mRNAs of the reconstructed lncRNA-mRNA interactions were mapped to the KEGG reference pathways, and then informative pathways were obtained. We used FDR to adjust the raw P-values by means of Benjamini-Hochberg procedure (21). We selected informative pathways using the criteria of FDR $<0.01$.

Establishment of condition-specific LCRP and hubs selection. Then, $\mathrm{R}$ package was used to transform the identified informative pathways above into undirected graphs which kept the structure of the original pathway (12). The lncRNAs in the lncRNA-mRNA interactions reweighted by PCC were aligned into the pathway graphs, which were as nodes via connecting to the mRNAs regulated by lncRNAs. Ultimately, the conditionspecific LCRP were constructed, which contained lncRNA nodes as well as IncRNA-mRNA competitively regulated edges.

To our knowledge, hubs were frequently considered important within the biological network. Therefore, in order to extract significant IncRNAs, we concentrated on the hub lncRNAs in the LCRP. In this study, the top $10 \%$ of lncRNAs having the highest degree of connectivity were extracted and determined as the hub lncRNAs.

Positioning sub-pathways competing regulated by lncRNAs. LncRNAs were considered as signature nodes, since these lncRNAs referred to the genes of interest and competing regulation. Integration of these lncRNAs with the topology features of LCRP is beneficial to effectively locate lncRNAmediated subregions. The IncRNAs were embedded in the LCRP, and then 'lenient distance' similarity was used to identify the lncRNA-regulated sub-pathways based on the topology characteristics of the LCRP. In the current study, $n$ (the number of molecules) $=1$ and $s$ (the number of nodes in the molecules in a pathway) $=8$ were employed to extract the candidate sub-pathways.

Assessment of significance of candidate sub-pathway. Wallenius approximation approach was applied to assess the 
Table I. The seed pathways.

\begin{tabular}{|c|c|}
\hline Pathways & $\begin{array}{l}\text { False discovery } \\
\text { rate (FDR) }\end{array}$ \\
\hline hsa05211: Renal cell carcinoma & $8.81 \mathrm{E}-05$ \\
\hline hsa04066: HIF-1 signaling pathway & 7.99E-05 \\
\hline hsa05169: Epstein-Barr virus infection & 7.13E-08 \\
\hline hsa05210: Colorectal cancer & 7.07E-06 \\
\hline hsa05215: Prostate cancer & $5.94 \mathrm{E}-11$ \\
\hline hsa05219: Bladder cancer & $5.44 \mathrm{E}-09$ \\
\hline hsa05220: Chronic myeloid leukemia & 4.75E-08 \\
\hline hsa04110: Cell cycle & $4.56 \mathrm{E}-07$ \\
\hline hsa04510: Focal adhesion & 4.45E-05 \\
\hline hsa04012: ErbB signaling pathway & $4.20 \mathrm{E}-08$ \\
\hline hsa05200: Pathways in cancer & $3.64 \mathrm{E}-10$ \\
\hline hsa05222: Small cell lung cancer & 3.64E-08 \\
\hline hsa05218: Melanoma & $3.31 \mathrm{E}-07$ \\
\hline hsa05223: Non-small cell lung cancer & 2.92E-07 \\
\hline hsa04151: PI3K-Akt signaling pathway & $2.81 \mathrm{E}-10$ \\
\hline hsa05203: Viral carcinogenesis & 2.32E-06 \\
\hline hsa04115: p53 signaling pathway & 2.09E-07 \\
\hline $\begin{array}{l}\text { hsa05120: Epithelial cell signaling in } \\
\text { Helicobacter pylori infection }\end{array}$ & $1.90 \mathrm{E}-06$ \\
\hline hsa05161: Hepatitis B & $1.55 \mathrm{E}-08$ \\
\hline hsa05212: Pancreatic cancer & $1.52 \mathrm{E}-07$ \\
\hline hsa05213: Endometrial cancer & $1.50 \mathrm{E}-05$ \\
\hline $\begin{array}{l}\text { hsa04722: Neurotrophin signaling } \\
\text { pathway }\end{array}$ & $1.12 \mathrm{E}-05$ \\
\hline hsa05214: Glioma & $1.02 \mathrm{E}-09$ \\
\hline hsa04150: mTOR signaling pathway & 9.92E-03 \\
\hline $\begin{array}{l}\text { hsa04620: Toll-like receptor signaling } \\
\text { pathway }\end{array}$ & $8.55 \mathrm{E}-03$ \\
\hline $\begin{array}{l}\text { hsa04810: Regulation of actin } \\
\text { cytoskeleton }\end{array}$ & $7.55 \mathrm{E}-03$ \\
\hline hsa05133: Pertussis & $5.90 \mathrm{E}-03$ \\
\hline hsa05152: Tuberculosis & $5.67 \mathrm{E}-03$ \\
\hline hsa04520: Adherens junction & $5.17 \mathrm{E}-03$ \\
\hline $\begin{array}{l}\text { hsa04662: B cell receptor signaling } \\
\text { pathway }\end{array}$ & 4.83E-03 \\
\hline $\begin{array}{l}\text { hsa04666: Fc gamma R-mediated } \\
\text { phagocytosis }\end{array}$ & $3.73 \mathrm{E}-03$ \\
\hline hsa04540: Gap junction & $3.29 \mathrm{E}-03$ \\
\hline $\begin{array}{l}\text { hsa04914: Progesterone-mediated } \\
\text { oocyte maturation }\end{array}$ & 3.09E-03 \\
\hline hsa05162: Measles & 2.66E-03 \\
\hline hsa05160: Hepatitis C & $2.52 \mathrm{E}-03$ \\
\hline $\begin{array}{l}\text { hsa03008: Ribosome biogenesis } \\
\text { in eukaryotes }\end{array}$ & 2.37E-03 \\
\hline hsa04740: Olfactory transduction & $2.36 \mathrm{E}-03$ \\
\hline hsa04728: Dopaminergic synapse & $2.28 \mathrm{E}-03$ \\
\hline hsa04730: Long-term depression & $1.92 \mathrm{E}-03$ \\
\hline hsa04350: TGF- $\beta$ signaling pathway & $1.79 \mathrm{E}-03$ \\
\hline hsa05166: HTLV-I infection & $1.66 \mathrm{E}-03$ \\
\hline hsa04910: Insulin signaling pathway & 9.71E-04 \\
\hline
\end{tabular}

Table I. Continued.

\begin{tabular}{lc}
\hline Pathways & $\begin{array}{c}\text { False discovery } \\
\text { rate (FDR) }\end{array}$ \\
\hline hsa04912: GnRH signaling pathway & $8.83 \mathrm{E}-04$ \\
hsa04062: Chemokine signaling pathway & $8.78 \mathrm{E}-04$ \\
hsa05168: Herpes simplex infection & $7.27 \mathrm{E}-04$ \\
hsa04720: Long-term potentiation & $6.21 \mathrm{E}-04$ \\
hsa04330: Notch signaling pathway & $5.84 \mathrm{E}-04$ \\
hsa04660: T cell receptor signaling & $4.37 \mathrm{E}-04$ \\
pathway & \\
hsa05142: Chagas disease (American & $4.37 \mathrm{E}-04$ \\
trypanosomiasis) & \\
hsa05216: Thyroid cancer & $3.72 \mathrm{E}-04$ \\
hsa05145: Toxoplasmosis & $2.67 \mathrm{E}-04$ \\
hsa04310: Wnt signaling pathway & $2.38 \mathrm{E}-04$ \\
hsa04621: NOD-like receptor signaling & $2.27 \mathrm{E}-04$ \\
pathway & \\
hsa05221: Acute myeloid leukemia & $2.27 \mathrm{E}-04$ \\
hsa04725: Cholinergic synapse & $1.74 \mathrm{E}-04$ \\
hsa04010: MAPK signaling pathway & $1.52 \mathrm{E}-04$ \\
\hline
\end{tabular}

significance of candidate sub-pathways based on BiasedUrn of $R$ package (22), to further evaluate whether the candidate sub-pathways were competitively regulated by 1 ncRNAs. During the evaluation process, the parameters below were used: the count of mRNAs of interest; the count of background mRNAs; the count of background mRNAs in a given sub-pathways; the number of interesting mRNAs enriched in this given sub-pathway; and the weight score of the sub-pathway. The weight was the strength of competing regulation by lncRNAs locating in this sub-pathway, which was computed using the equation described in a previous study (11). Next, FDR was used to correct the original P-values, and the significant sub-pathways were selected when on the threshold of FDR was set as 0.01 .

\section{Results}

Co-expression analysis for IncRNA-mRNA interactions and informative pathways identification. Before identifying informative pathways, PCC was used to evaluate the co-expression possibility for IncRNA-mRNA interactions according to matched lncRNA as well as mRNA data. When the criteria were set at a $\mathrm{P}<0.05$, overall 44 lncRNAs, 165 mRNAs and 224 co-expressed interactions were extracted (data not shown). In order to detect seed pathways, these 165 mRNAs were then embedded into the background pathways. Finally, we obtained a total of 56 informative pathways when the FDR was set at 0.01 (Table I). Of note, the three most significant pathways were PI3K-Akt signaling pathway (FDR=2.81E-10), pathways in cancer $(\mathrm{FDR}=3.64 \mathrm{E}-10)$, and glioma $(\mathrm{FDR}=1.02 \mathrm{E}-09)$.

Selecting lncRNA-regulated sub-pathways. Then, the 56 informative pathways were embedded in undirected graphs, 


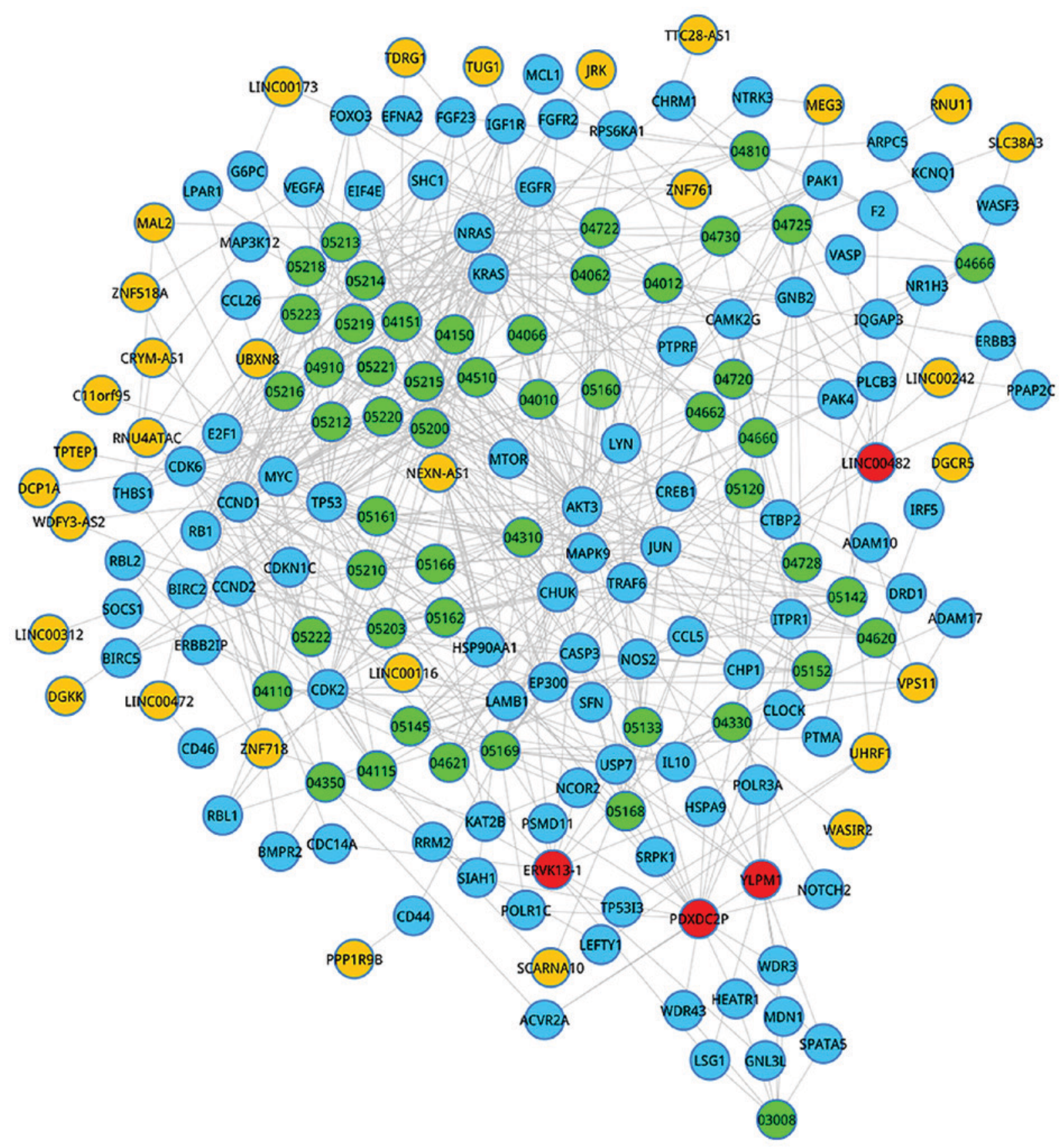

Figure 1. Construction of condition-specific lncRNA competitively regulated pathways (LCRP) on the basis of matched lncRNA data, mRNA data and potential lncRNA-mRNA interactions. Yellow, blue, and green nodes represent lncRNAs, mRNAs, and pathways, respectively. Red nodes are hub lncRNAs.

and the 44 lncRNAs were mapped to the pathway graphs as nodes. After that, a condition-specific LCRP was built (Fig. 1). To extract crucial lncRNAs of heart failure, a degree analysis was implemented for all nodes of the LCRP. According to degree distribution, a total of 4 hub lncRNAs were selected, including ERVK13-1, YLPM1, PDXDC2P, and LINC00482.

Based on the information of the LCRP, we discovered a total of 36 lncRNAs competitively regulating sub-pathways involved in 40 complete pathways when the FDR was $<0.01$ (Table II). In advanced investigation, we mainly concentrated on the top three sub-pathways (Fig. 2). The first most significant sub-pathway was path: 04010_1, which was a subpart of MAPK signaling pathway (Fig. 2A). Subsequently, we further analyzed this sub-pathway, and we found that a total of 13 IncRNAs competitively regulated this subpart synergisti- cally. Within these lncRNAs, two lncRNAs (PDXDC2P and NEXN-AS1), directly regulated three genes. In addition, MYC was coordinately regulated by three lncRNAs (CRYM-AS1, MAL2, and UBXN8). Moreover, AKT3 was also simultaneously regulated by three lncRNAs (ERVK13-1, YLPM1, and PDXDC2P).

The second significant subregion was path: 04012-1, an important subpart in ErbB signaling pathway (Fig. 2B). Similar to the first significant sub-pathway, gene AKT3 was also simultaneously regulated by three lncRNAs (ERVK13-1, YLPM1, and PDXDC2P).

The third sub-pathway, path: 04062_2, was a part of chemokine signaling pathway (Fig. 2C). Similar to the first and the second significant sub-pathways, gene AKT3 was also simultaneously regulated by three lncRNAs (ERVK13-1, 

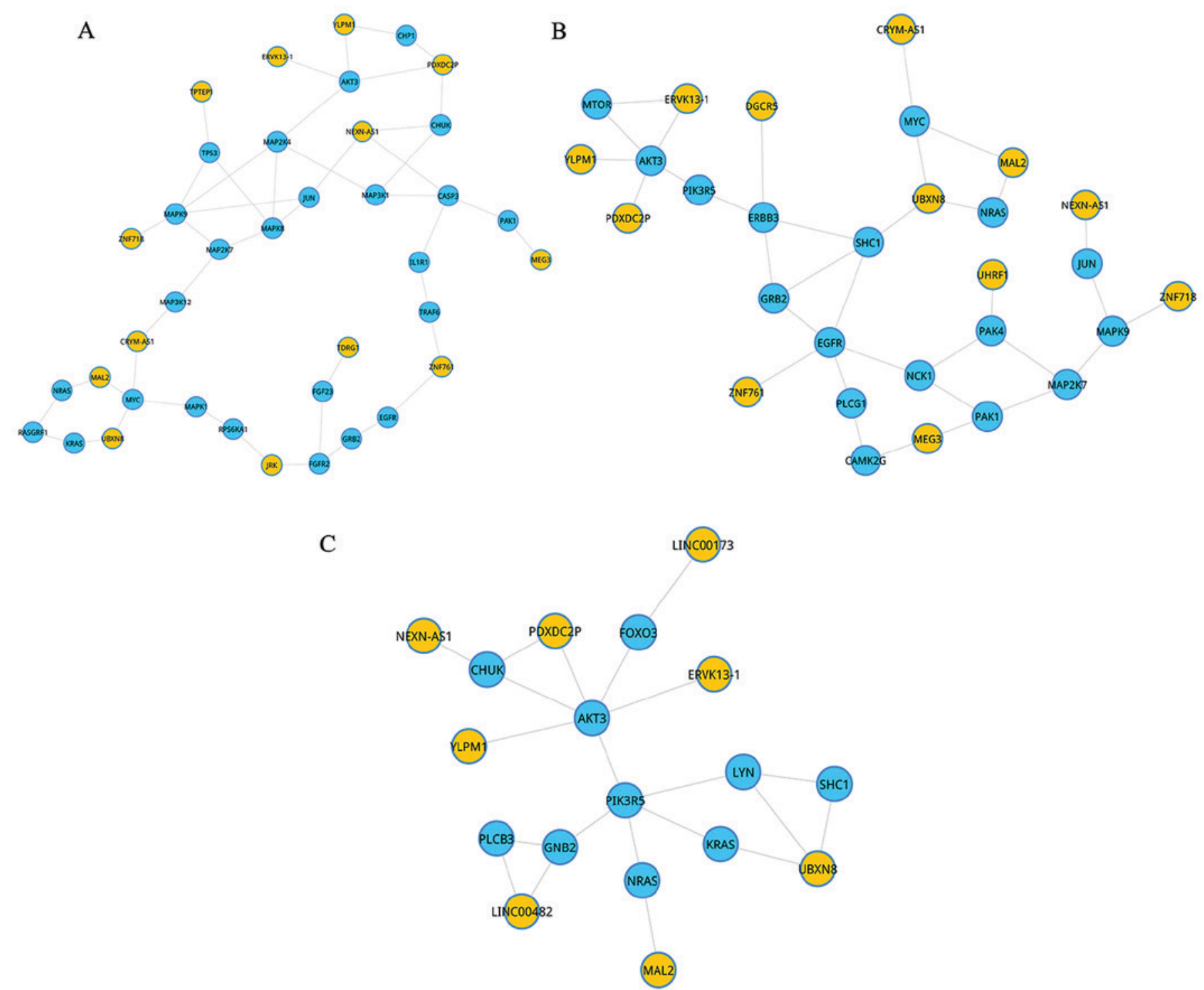

Figure 2. Sub-pathways identified using the sub-pathway strategy. Yellow and blue nodes stand for lncRNAs and mRNAs, respectively. (A) MAPK signaling pathway (path: 04010_1, FDR=2.80E-16). (B) ErbB signaling pathway (path: 04012_1, FDR=5.35E-16). (C) Chemokine signaling pathway (path: 04062_2, $F D R=1.96 \mathrm{E}-15)$.

YLPM1, and PDXDC2P). Notably, IncRNA UBXN8 competitively regulated three genes (KRAS, LYN, and SHC1). Thus, these results demonstrated that this approach could identify biological meaningful sub-pathways, and highlighted some critical lncRNAs in heart failure condition.

\section{Discussion}

Currently, alteration of lncRNA competitively regulated functions may result in the occurrence and progression of diseases, but, a better understanding of this regulatory mechanism may give more and better opportunities for developing new therapies. Hence we constructed an LCRP for the informative pathways, and integrated the lncRNAs with the topology features of LCRP. Normally, comparing with the entire pathways, sub-pathways may be more explainable to the molecular mechanisms underlying disorders. Consequently, detecting the sub-pathways which were regarded to be more possibly disrupted pathways from the LCRP would be the best means to discriminate the heart failure group from the control group.

Thus, an LCRP was constructed with 36 lncRNAs, 102 mRNA nodes and 40 pathways by combining gene expression profile, IncRNA data, pathway information and PCC related analyses. Finally, based on the information of the LCRP, a total of 36 lncRNAs competitively regulating sub-pathways involved in 40 complete pathways were identified. Among these 40 pathways, we mainly concentrated on the top three sub-pathways that competitively regulated by lncRNAs, including path: 04010_1, which was a subpart of MAPK signaling pathway, path: 04012-1, an important subpart in ErbB signaling pathway, and path: 04062_2, a part of chemokine signaling pathway.

Taking MAPK signaling pathways as examples to discuss, the MAPK signaling pathway has been implicated to control myocyte growth that responds to pathological stimuli in the heart (23). Heart failure is considered to be a remodeling process of myocyte cells, and cellular as well as extracellular 
Table II. Sub-pathways list based on FDR $<0.01$

\begin{tabular}{|c|c|c|}
\hline Pathway IDs & Pathway name & FDR \\
\hline 04010_1 & MAPK signaling pathway & $2.80 \mathrm{E}-16$ \\
\hline 04012_1 & ErbB signaling pathway & $5.35 \mathrm{E}-16$ \\
\hline 04062_2 & Chemokine signaling pathway & $1.96 \mathrm{E}-15$ \\
\hline 04110_1 & Cell cycle & $5.16 \mathrm{E}-15$ \\
\hline $04115 \_1$ & p53 signaling pathway & $4.50 \mathrm{E}-15$ \\
\hline 04151_1 & PI3K-Akt signaling pathway & $4.53 \mathrm{E}-15$ \\
\hline 04310_1 & Wnt signaling pathway & $4.62 \mathrm{E}-15$ \\
\hline 04510_1 & Focal adhesion & $4.87 \mathrm{E}-15$ \\
\hline 04722_1 & Neurotrophin signaling pathway & $1.88 \mathrm{E}-14$ \\
\hline 05161_1 & Hepatitis B & $3.28 \mathrm{E}-14$ \\
\hline 05166_1 & HTLV-I infection & $5.96 \mathrm{E}-14$ \\
\hline 05168_1 & Herpes simplex infection & $7.40 \mathrm{E}-14$ \\
\hline 05169_1 & Epstein-Barr virus infection & $1.56 \mathrm{E}-13$ \\
\hline 05200_1 & Pathways in cancer & $5.33 \mathrm{E}-13$ \\
\hline 05212_1 & Pancreatic cancer & $5.92 \mathrm{E}-13$ \\
\hline 05214_1 & Glioma & $2.06 \mathrm{E}-12$ \\
\hline 05215_1 & Prostate cancer & $5.99 \mathrm{E}-12$ \\
\hline 05218_1 & Melanoma & $6.87 \mathrm{E}-11$ \\
\hline 05222_1 & Small cell lung cancer & $6.85 \mathrm{E}-11$ \\
\hline 05223_1 & Non-small cell lung cancer & $2.16 \mathrm{E}-10$ \\
\hline 04728_1 & Dopaminergic synapse & $5.61 \mathrm{E}-10$ \\
\hline 05203_2 & Viral carcinogenesis & $7.29 \mathrm{E}-10$ \\
\hline 04066_1 & HIF-1 signaling pathway & $1.61 \mathrm{E}-09$ \\
\hline 05152_2 & Tuberculosis & $2.54 \mathrm{E}-09$ \\
\hline 04720_1 & Long-term potentiation & $7.51 \mathrm{E}-09$ \\
\hline 04810_3 & Regulation of actin cytoskeleton & $8.92 \mathrm{E}-09$ \\
\hline 04662_1 & B cell receptor signaling pathway & $2.21 \mathrm{E}-08$ \\
\hline 05145_1 & Toxoplasmosis & $3.08 \mathrm{E}-08$ \\
\hline 03008_1 & Ribosome biogenesis in eukaryotes & 7.25E-08 \\
\hline 05210_1 & Colorectal cancer & $1.79 \mathrm{E}-07$ \\
\hline 05220_1 & Chronic myeloid leukemia & $9.38 \mathrm{E}-07$ \\
\hline 05221_1 & Acute myeloid leukemia & 2.29E-06 \\
\hline 04620_1 & Toll-like receptor signaling pathway & $1.61 \mathrm{E}-06$ \\
\hline 04330_1 & Notch signaling pathway & $3.67 \mathrm{E}-06$ \\
\hline 04660_2 & $\mathrm{T}$ cell receptor signaling pathway & $8.54 \mathrm{E}-06$ \\
\hline 05213_1 & Endometrial cancer & $9.12 \mathrm{E}-06$ \\
\hline 04910_1 & Insulin signaling pathway & $3.27 \mathrm{E}-05$ \\
\hline 05160_1 & Hepatitis C & $1.59 \mathrm{E}-05$ \\
\hline 05162_2 & Measles & $9.14 \mathrm{E}-05$ \\
\hline 04350_1 & TGF- $\beta$ signaling pathway & $1.09 \mathrm{E}-04$ \\
\hline
\end{tabular}

factors participate in this remodeling process to further cause the changes in myocardial structures and functions. Matrix metalloproteinases (MMPs) are the factors participating in this remodeling process. Significantly, MMPs secretion has been reported to be dependent on MAPK or MAPK signaling pathway $(24,25)$. Thus, we speculate that a close relationship exists between the heart failure and MAPK signaling pathway.
Of note, in the first, second, and third most significant sub-pathways, gene AKT3 was simultaneously regulated by three lncRNAs (ERVK13-1, YLPM1, and PDXDC2P). Moreover, ERVK13-1, YLPM1, and PDXDC2P were all hubs in the LCRP. YLPM1 was also termed as ZAP3. A former study has demonstrated that the highest expression of ZAP3 seems to be in brain, heart and kidneys (26). Of note, ZAP3 has been implicated to decrease telomerase activity (27). Further, telomerase ablation can cause the occurrence of heart failure (28). Thus, we infer that lncRNA YLPM1 might play crucial roles in the development of heart failure, partially via regulating the telomerase activity. Although few reports have suggested the link between lncRNAs (ERVK13-1, or PDXDC2P) and heart failure, we found these lncRNAs regulated the common gene AKT3 in our study. AKT3 is one of the three isoforms of AKT which mediates cardiac growth, cell death, and myocardial angiogenesis in cardiac myocytes (29). Moreover, coronary vasculature, and cell death are essential for the maintenance of the contractile function, especially in heart failure (30). Accordingly, we infer that lncRNAs (ERVK13-1 and PDXDC2P) have indirect relationships with heart failure.

The current study had several limitations. The study population was small. The lack of verification of microarray results by means of quantitative real-time PCR technique or western blot based on animal or patient tissues was another important drawback. Thus, further studies in larger study samples are necessary to confirm these findings in terms of the efficiency as diagnostic or prognostic biomarkers of heart failure.

In conclusion, we applied sub-pathway-based analysis by introducing the concept of pathway topology, lncRNA data, and subregion of pathways. The importance of this method is that it can help to understand the etiology of diseases, and provide important insight into the functions of lncRNAs in heart failure. Based on the results, we showed that MAPK signaling pathway, ErbB signaling pathway, as well as chemokine signaling pathway were the significant sub-pathways for heart failure. Nevertheless, the verification using other datasets is implemented to demonstrate that these sub-pathways are useful in classifying heart failure and normal group.

\section{Acknowledgements}

Not applicable.

\section{Funding}

No funding was received.

\section{Availability of data and materials}

The datasets used and/or analyzed during the current study are available from the corresponding author on reasonable request.

\section{Authors' contributions}

DMH conceived the study, analyzed the data and drafted the manuscript. The author has read and approved the final manuscript. 


\section{Ethics approval and consent to participate}

Not applicable.

\section{Patient consent for publication}

Not applicable.

\section{Competing interests}

The author declares that he has no competing interests.

\section{References}

1. McMurray JJ and Pfeffer MA: Heart failure. Lancet 365: 1877-1889, 2005.

2. Charlson FJ, Erskine HE, Ferrari AJ, Leung J, Whiteford HA, Abajobir AA, Knibbs LD, Lalloo R, Scott JG and Guo Y; GBD 2015 Disease and Injury Incidence and Prevalence Collaborators: Global, regional, and national incidence, prevalence, and years lived with disability for 310 diseases and injuries, 1990-2015: A systematic analysis for the Global Burden of Disease Study 2015. Lancet 388: 1545-1602, 2016.

3. Go AS, Mozaffarian D, Roger VL, Benjamin EJ, Berry JD, Borden WB, Bravata DM, Dai S, Ford ES, Fox CS, et al American Heart Association Statistics Committee and Stroke Statistics Subcommittee: Executive summary: heart disease and stroke statistics - 2013 update: a report from the American Heart Association. Circulation 127: 143-152, 2013.

4. Kusumoto A, Miyata M, Kubozono T, Ikeda Y, Shinsato T, Kuwahata S, Fujita S, Takasaki K, Yuasa T, Hamasaki S, et al: Highly sensitive cardiac troponin $\mathrm{T}$ in heart failure: Comparison with echocardiographic parameters and natriuretic peptides. J Cardiol 59: 202-208, 2012

5. Di Gesualdo F, Capaccioli S and Lulli M: A pathophysiological view of the long non-coding RNA world. Oncotarget 5: 10976-10996, 2014

6. Zhao W, Luo J and Jiao S: Comprehensive characterization of cancer subtype associated long non-coding RNAs and their clinical implications. Sci Rep 4: 6591, 2014

7. Gomes da Silva AM and Silbiger VN: miRNAs as biomarkers of atrial fibrillation. Biomarkers 19: 631-636, 2014.

8. Liang WC, Fu WM, Wong CW, Wang Y, Wang WM, Hu GX, Zhang L, Xiao LJ, Wan DC, Zhang JF, et al: The lncRNA H19 promotes epithelial to mesenchymal transition by functioning as miRNA sponges in colorectal cancer. Oncotarget 6: 22513-22525, 2015.

9. Papait R, Kunderfranco P, Stirparo GG, Latronico MVG and Condorelli G: Long noncoding RNA: A new player of heart failure? J Cardiovasc Transl Res 6: 876-883, 2013.

10. Han P, Li W, Lin CH, Yang J, Shang C, Nuernberg ST, Jin KK, $\mathrm{Xu} \mathrm{W}$, Lin CY, Lin CJ, et al: A long noncoding RNA protects the heart from pathological hypertrophy. Nature 514: 102-106, 2014.

11. Shi X, Xu Y, Zhang C, Feng L, Sun Z, Han J, Su F, Zhang Y, $\mathrm{Li} \mathrm{C}$ and Li X: Subpathway-LNCE: Identify dysfunctional subpathways competitively regulated by lncRNAs through integrating lncRNA-mRNA expression profile and pathway topologies. Oncotarget 7: 69857-69870, 2016.

12. Li C, Han J, Yao Q, Zou C, Xu Y, Zhang C, Shang D, Zhou L, Zou C, Sun Z, et al: Subpathway-GM: Identification of metabolic subpathways via joint power of interesting genes and metabolites and their topologies within pathways. Nucleic Acids Res 41: e101, 2013.
13. Li X, Shen L, Shang X and Liu W: Subpathway analysis based on signaling-pathway impact analysis of signaling pathway. PLoS One 10: e0132813, 2015

14. Greco S, Fasanaro P, Castelvecchio S, D'Alessandra Y, Arcelli D, Di Donato M, Malavazos A, Capogrossi MC, Menicanti L and Martelli F: MicroRNA dysregulation in diabetic ischemic heart failure patients. Diabetes 61: 1633-1641, 2012.

15. Irizarry RA, Bolstad BM, Collin F, Cope LM, Hobbs B and Speed TP: Summaries of Affymetrix GeneChip probe level data. Nucleic Acids Res 31: e15, 2003.

16. Bolstad BM, Irizarry RA, Astrand M and Speed TP: A comparison of normalization methods for high density oligonucleotide array data based on variance and bias. Bioinformatics 19: 185-193, 2003.

17. Wang HB, Jiang ZB and Li M: Research on the typical miRNA and target genes in squamous cell carcinoma and adenocarcinoma of esophagus cancer with DNA microarray. Pathol Oncol Res 20: 245-252, 2014

18. Nahler G: Pearson correlation coefficient. In: Dictionary of Pharmaceutical Medicine. Springer-Verlag Vienna, Austria, p132, 2009.

19. Best DJ and Roberts DE: Algorithm AS 89: The upper tail probabilities of Spearman's Rho. Appl Stat 24: 377-379, 1975.

20. Kanehisa M, Araki M, Goto S, Hattori M, Hirakawa M, Itoh M, Katayama T, Kawashima S, Okuda S, Tokimatsu T, et al: KEGG for linking genomes to life and the environment. Nucleic Acids Res 36: D480-D484, 2008.

21. Benjamini Y and Hochberg Y: Controlling the false discovery rate: A practical and powerful approach to multiple testing. J R Stat Soc B 57: 289-300, 1995

22. Epstein MP, Duncan R, Jiang Y, Conneely KN, Allen AS and Satten GA: A permutation procedure to correct for confounders in case-control studies, including tests of rare variation. Am J Hum Genet 91: 215-223, 2012.

23. Molkentin JD and Dorn GW II: Cytoplasmic signaling pathways that regulate cardiac hypertrophy. Annu Rev Physiol 63: 391-426, 2001.

24. Kim ES, Kim MS and Moon A: TGF-beta-induced upregulation of MMP-2 and MMP-9 depends on p38 MAPK, but not ERK signaling in MCF10A human breast epithelial cells. Int J Oncol 25: 1375-1382, 2004

25. Qiu Q, Yang M, Tsang BK and Gruslin A: EGF-induced trophoblast secretion of MMP-9 and TIMP-1 involves activation of both PI3K and MAPK signalling pathways. Reproduction 128: 355-363, 2004.

26. Ulke-Lemée A, Trinkle-Mulcahy L, Chaulk S, Bernstein NK, Morrice N, Glover M, Lamond AI and Moorhead GBG: The nuclear PP1 interacting protein ZAP3 (ZAP) is a putative nucleoside kinase that complexes with SAM68, CIA, NF110/45, and HNRNP-G. Biochim Biophys Acta 1774: 1339-1350, 2007.

27. Armstrong L, Lako M, van Herpe I, Evans J, Saretzki G and Hole $\mathrm{N}$ : A role for nucleoprotein Zap3 in the reduction of telomerase activity during embryonic stem cell differentiation. Mech Dev 121: 1509-1522, 2004

28. Leri A, Franco S, Zacheo A, Barlucchi L, Chimenti S, Limana F, Nadal-Ginard B, Kajstura J, Anversa P and Blasco MA: Ablation of telomerase and telomere loss leads to cardiac dilatation and heart failure associated with p53 upregulation. EMBO J 22: 131-139, 2003.

29. Shiojima I and Walsh K: Regulation of cardiac growth and coronary angiogenesis by the Akt/PKB signaling pathway. Genes Dev 20: 3347-3365, 2006.

30. Chaanine AH and Hajjar RJ: AKT signalling in the failing heart. Eur J Heart Fail 13: 825-829, 2011.

(i) $\ominus$ This work is licensed under a Creative Commons cc) ${ }_{\mathrm{EY}}$ NO ND Attribution-NonCommercial-NoDerivatives 4.0 International (CC BY-NC-ND 4.0) License. 